\title{
Tests de croissance de jeunes plants de néré (Parkia biglobosa, Jack, R. Br.) en pépinière
}

\author{
P. C. GNANGLE ${ }^{1}$, R. GLELE KAKAÏ ${ }^{2 *}$, M. OUMOROU ${ }^{3}$, K. N'DJOLOSSE ${ }^{1}$, \\ W. BONOU ${ }^{2}$ et N. SOKPON ${ }^{4}$. \\ ${ }^{1}$ INRAB / CRA-Centre, PRF, BP 112 Savè, Bénin. E-mail: gnampaces@yahoo.fr \\ ${ }^{2}$ Faculté des sciences agronomiques, Université d'Abomey-Calavi, 01 BP 526 Cotonou, Bénin. \\ ${ }^{3}$ Ecole Polytechnique d'Abomey-Calavi, Université d'Abomey-Calavi, 01 BP 2009 Cotonou, Bénin. \\ ${ }^{4}$ Faculté d'agronomie, Université de Parakou, BP 123, Bénin. \\ *Auteur correspondant, E-mail: glele.romain@gmail.com
}

\section{RESUME}

Cinq accélérateurs de croissance incluant l'engrais et la période d'application d'extraits aqueux de Moringa oleifera ont été testés sur 1288 jeunes plants de 15 provenances de néré (Parkia biglobosa) pour évaluer leur croissance morphologique. Les provenances étaient issues de 15 villages échantillonnés dans les 5 parcs à karité-néré du Bénin. L'essai a été conduit en station suivant un dispositif complètement aléatoire à 3 répétitions. Une analyse de variance a été réalisée sur les données morphologiques des plants au $140^{\text {ème }}$ jour après semis et sur l'évolution de ceux-ci dans le temps afin de tester l'effet des provenances et des traitements appliqués. Les résultats obtenus ont révélé que la performance des provenances de néré n'était pas influencée par les conditions climatiques de la zone de prélèvement des semences. En l'absence d'engrais, la provenance P6 (Savè) a donné les meilleurs résultats $(6,6 \mathrm{~cm}$ de diamètre au collet, $26,3 \mathrm{~cm}$ de hauteur totale et 8,5 feuilles au $140^{\text {ème }}$ jour après semis). Avec l'application d'engrais, les provenances P1, P2, P3, P4, P6, P8, P10 et P13 présentent les valeurs les plus élevées des paramètres. L'application d'extraits aqueux de feuilles de $M$. oleifera tous les 10 jours a donné des résultats satisfaisants. Ces résultats suggèrent la considération des arbres-élites dans les tests de provenance de l'espèce.

(C) 2010 International Formulae Group. All rights reserved.

Mots clés: Parkia biglobosa Jack. R. Br, Provenances, accélérateur de croissance, Moringa oleifera, croissance morphologique.

\section{INTRODUCTION}

Les forêts africaines constituent un immense réservoir de diversité biologique et jouent un rôle fondamental dans la satisfaction de nombreux besoins des populations locales (IPGRI, 1999). Au Bénin, environ 175 espèces végétales forestières sont identifiées et consommées à travers leurs feuilles, leurs fruits, leurs graines, leurs racines, leurs tubercules et leurs fleurs (Codjia et al., 2001). Parmi celles-ci figure le néré (Parkia biglobosa, Jack, R. Br.), l'une des espèces alimentaires dont l'importance est bien reconnue tant au niveau régional qu'international comme en témoignent les recommandations et les plans d'action du 
groupe d'experts de la FAO sur les ressources forestières génétiques et du Programme Sahélien de Semences Forestières (Ouédraogo, 1995).

Plusieurs utilisations du néré ont été recensées. En effet, les produits du néré contribuent à la complémentation alimentaire familiale et nationale en créant des ressources utilisables directement pour se procurer de la nourriture ou pour développer les systèmes de production alimentaire. Ils sont nécessaires pour augmenter l'apport protidique du régime alimentaire des populations rurales tropicales, constitué essentiellement de céréales. Ceci permet de réduire le déséquilibre alimentaire en protéines et en sucres et d'améliorer la santé des populations (Malaisse, 1997). Les apports nutritifs des graines de néré fermentées comparées à ceux de la viande de poulet révèlent que, pour $40 \mathrm{~kg}$ de néré, il faudrait 46 poulets pour avoir autant de protéines, 61 pour avoir autant de graisses et 57 pour avoir autant de calories que la production de soumbala obtenue par transformation (Dupriez et De Leener, 1993). Par ailleurs, l'espèce serait aussi utilisée en médecine traditionnelle comme recette principale ou en association avec d'autres plantes dans les soins de plusieurs affections en Afrique de l'Ouest (Lejoly, 1990 ; Sokpon et Ouinsavi, 2004; Koura, 2005). Ces affections seraient pour la plupart les plaies par brûlures, les dermatoses, les oedèmes, les bronchites, la dysenterie, la stérilité, l'oreillon et la pneumonie (CIRAD-GRET-MAE, 2002). En plus, le néré est une légumineuse et contribue ainsi à la restauration et à l'amélioration de la fertilité des sols grâce à sa capacité fixatrice d'azote (Dupriez et De Leener, 1993). Au Bénin, elle constitue avec le karité (Vitellaria paradoxa, Gaertn), les espèces ligneuses les plus utilisées dans les parcs et systèmes agroforestiers traditionnels (Agbahungba et Depommier, 1989 ; Sokpon, 1994). Dans l'économie rurale, le néré est aussi utilisé pour la pêche, l'aviculture, l'alimentation du bétail, l'artisanat, la construction (Ouédraogo, 1995). Il constitue une importante source de revenu pour les populations qui font la commercialisation de ses fruits (Gutierrez, 2000).

Cependant, dans la plupart des pays de l'Afrique de l'Ouest, l'expansion de l'agriculture, conséquence de la croissance démographique, a sensiblement réduit et transformé l'habitat du néré en des parcs agroforestiers avec des densités relativement faibles de l'espèce, variant entre 1 et 6 arbres/ha (Gbédji, 2003). Au Bénin, les populations du néré ne sont plus systématiquement épargnées lors des défrichements et les parcs à néré sont caractérisés par une forte présence d'arbres relativement vieux, révélant une faible régénération des peuplements et une forte régression de l'aire de répartition (Gijsbers et al., 1994 ; Ouédraogo, 1995 ; Sambe et al., 2010). Par ailleurs, le néré est resté jusqu'à présent, dans la plupart des zones favorables à sa culture, à l'état sauvage et demeure encore un produit de cueillette. L'utilisation de son bois pour la fabrication de mortier et du charbon de bois dans certaines régions du Bénin contribue fortement à sa raréfaction.

La domestication des espèces forestières alimentaires comme le néré, menacées dans leurs aires de répartition devient donc une nécessité afin d'accroître le bien-être des populations rurales africaines (Leakey et al., 2005). Toute démarche de domestication débute par l'exploitation de la variabilité naturelle existante en sélectionnant avec l'aide des populations, les sujets aux phénotypes les plus intéressants pour les critères considérés (Soloviev et al., 2004). L'étape suivante vise à mettre au point des techniques de propagation végétative permettant de fixer les caractéristiques génétiques du matériel végétal sélectionné (Danthu et al., 1995) ainsi qu'à poursuivre l'évaluation du matériel végétal collecté. L'objectif final est de transférer aux populations rurales, des accessions sélectionnées pour leur productivité, leur qualité ainsi que leur résistance à divers ennemis et maladies, afin qu'elles les 
introduisent dans les parcs agroforestiers, dans les jardins de case ou dans les vergers. De ce fait, pour une meilleure connaissance des individus de néré encore existants dans les parcs, les meilleures provenances doivent être répertoriées en vue d'évaluer leurs performances phénotypiques pour leur domestication.

Cette étude avait pour objectif d'identifier les meilleures provenances de néré et de tester l'effet de différents accélérateurs de croissance incluant des extraits aqueux à base de Moringa oleifera sur de jeunes plants en pépinière. M. oleifera a en effet la propriété d'augmenter la vitesse de croissance et les rendements floraux des légumineuses (Foidl et al., 2001).

\section{MATERIEL ET METHODES}

Site expérimental et matériel végétal

Les semences de néré collectées dans les parcs à karité-néré ont été élevées en pépinière dans l'enceinte du siège du Centre de Recherche Agricole du Centre à Savè (Bénin). Ce site est caractérisé par une pluviométrie et une température moyenne de $1110 \mathrm{~mm}$ et de $27,6^{\circ} \mathrm{C}$, respectivement.

Le matériel végétal est constitué des semences de néré. Elles ont été collectées dans 15 villages issus des cinq parcs karité et néré identifiés au Bénin (Gbédji, 2003 ; Gnanglè, 2005). Ces parcs sont localisés entre la latitude de Bohicon $\left(7^{\circ} \mathrm{N}\right)$ et celle de Karimama $\left(12^{\circ} \mathrm{N}\right)$.

Dans chaque parc, les graines sont prélevées dans trois différents villages distants d'au moins $30 \mathrm{~km}$ au Sud et au Centre (Bohicon, Savè et Parakou) et d'au moins $50 \mathrm{~km}$ au Nord (Bembèrèkè et Kandi). Les caractéristiques des différentes provenances sont présentées au Tableau 1. On note de ce tableau que les trois premiers parcs sont situés dans le domaine soudano-guinéen et les deux derniers dans le domaine soudanien. Les lieux de collecte des graines de néré pour la mise en place de la pépinière sont indiqués à la Figure 1.

\section{Dispositif expérimental et collecte des données}

Les graines de néré ont été ensemencées dans des sacs en polyéthylène de $35 \mathrm{~cm} \times 25 \mathrm{~cm}$. Les pots contenant les semences ont été disposés suivant un dispositif complètement aléatoire à deux facteurs : la provenance et l'accélérateur de croissance, le site d'expérimentation étant relativement homogène. Le substrat sur lequel les graines ont été semées est constitué d'un terreau provenant d'un site de dépôt et de gestion des déchets ménagers à proximité du centre de recherche agricole. Il a ensuite été tamisé avant usage. Les pots ont été arrosés jusqu'à ce que le terreau soit à sa capacité au champ avant que le semis n'a été effectué. En pépinière, cinq différents traitements ou accélérateurs de croissance ont été testés au regard des paramètres de croissance des plants de néré. Comme on peut le noter du Tableau 2 , les accélérateurs de croissance appliqués sont l'absence d'accélérateur de croissance (témoin T0), la fumure avec l'engrais NPK 1423-14 (T1), l'aspersion foliaire tous les 10 jours d'un extrait aqueux de feuilles de Moringa oleifera (T10), l'aspersion foliaire tous les 20 jours d'un extrait aqueux de feuilles de Moringa oleifera (T20) et l'aspersion foliaire tous les 30 jours d'un extrait aqueux de feuilles de Moringa oleifera (T30). Des parcelles élémentaires de 6 pots de même provenance ont été considérées, chaque parcelle élémentaire reçevant un traitement donné et répété 3 fois.

La première aspersion foliaire d'extrait de feuilles de $M$. oleifera a été faite le 8 mai 2009. Les autres aspersions ont eu lieu tous les 10 jours et ce, pendant 4 mois. Les données collectées sont le diamètre au collet des plants, la hauteur totale des plants et le nombre de feuilles portées par les plants. Les mesures ont été réalisées à 50, 80, 110 et 140 jours après le semis pour le diamètre au collet et la hauteur totale des plants tandis qu'elles ont été réalisées pour le nombre de feuilles à 80,110 et 140 jours après le semis. 
Traitement statistique des données Appréciation de l'effet des traitements et provenances sur la morphologie des plants de néré

Afin d'analyser l'effet global des provenances et traitements d'accélération de croissance sur la morphologie des plants de néré à la fin de l'expérimentation (140 JAS), une analyse de la variance, modèle fixe à deux facteurs a été effectuée. Les valeurs moyennes ajustées des paramètres morphologiques des plants (diamètre au collet, hauteur des plants et nombre de feuilles) ont été d'abord calculées suivant chacun des 5 traitements et pour chaque provenance. Du fait du nombre élevé des niveaux du facteur «provenance» (15), une classification numérique sur les paramètres morphologiques a été effectuée pour chaque traitement (Dagnelie, 1998). Le but de cette analyse était d'identifier pour chaque traitement, les groupes homogènes de provenance suivant les paramètres morphologiques des jeunes plants de néré.

Appréciation de l'effet des traitements et provenances sur la croissance des plants de néré

Par ailleurs, pour tester l'effet des différents traitements sur chacun des paramètres morphologiques de croissance des provenances de néré tout en tenant compte des corrélations entre les mesures successives prises dans le temps, une analyse de la variance sur mesures répétées a été effectuée (Crowder et Hand, 1990). Le modèle utilisé est fixe à deux facteurs (provenances et traitements) et les données ont été traitées avec le logiciel SAS (SAS Inc., 2003).

Afin d'analyser la tendance évolutive des paramètres morphologiques des plants de néré suivant les provenances et traitements, des nuages de points représentant la variation de chaque paramètre morphologique en fonction du nombre de jours après la mise en pépinière ont été établis pour chaque paramètre. Ces nuages ont permis de noter globalement que la tendance d'évolution du diamètre au collet $(D c)$ et de la hauteur $(H t)$ des plants est exponentielle, de la forme:

$y\left(D_{c}\right.$ ou $\left.H_{t}\right)=b_{0} \exp \left(b_{1} N_{j}\right)$

Dans l'expression (1), $y$ est le paramètre de croissance mesuré ( $D c$ ou $H t$ ), $b_{0}$ représente les conditions initiales de croissance (valeur du paramètre en début d'application du traitement d'accélération de croissance) et $b_{1}$ est lié à la vitesse exponentielle de croissance du paramètre considéré. $N_{j}$ représente le nombre de jours après semis.

En ce qui concerne le nombre de feuilles, $\left(N_{f}\right)$, une tendance quadratique a été notée, dont l'équation est :

$$
N_{f}=b_{0}+b_{1} N_{j}+b_{2} N_{j}^{2}
$$

Dans la formule (2), $b_{0}$ représente le nombre initial de feuilles du plant considéré avant l'application des traitements d'accélérateur de croissance ; $b_{1}$ est la vitesse linéaire de croissance sachant que $b_{3}$ est la vitesse quadratique de croissance. Une extrapolation est faite jusqu'au $170^{\text {ème }}$ jour après semis afin de mieux conjecturer le comportement des feuilles le $140^{\text {ème }}$ jours après semis.

Pour chaque plant de néré, les coefficients $b_{0}, b_{1}$ et $b_{2}$ ont été estimés par la méthode des moindres carrés (Dagnelie, 1998). Les valeurs estimées de ces coefficients de régression ont été ensuite soumises à une analyse de la variance à deux facteurs (Provenance et Traitement) en utilisant le modèle fixe. L'interaction Provenance $\mathrm{x}$ traitement étant significative, une classification numérique a été réalisée sur les moyennes ajustées de chacun des coefficients $b_{0}, b_{1}$ et $b_{z}$ des différents paramètres morphologiques. Le but de cette analyse était de grouper pour chaque traitement appliqué, les provenances de néré. 


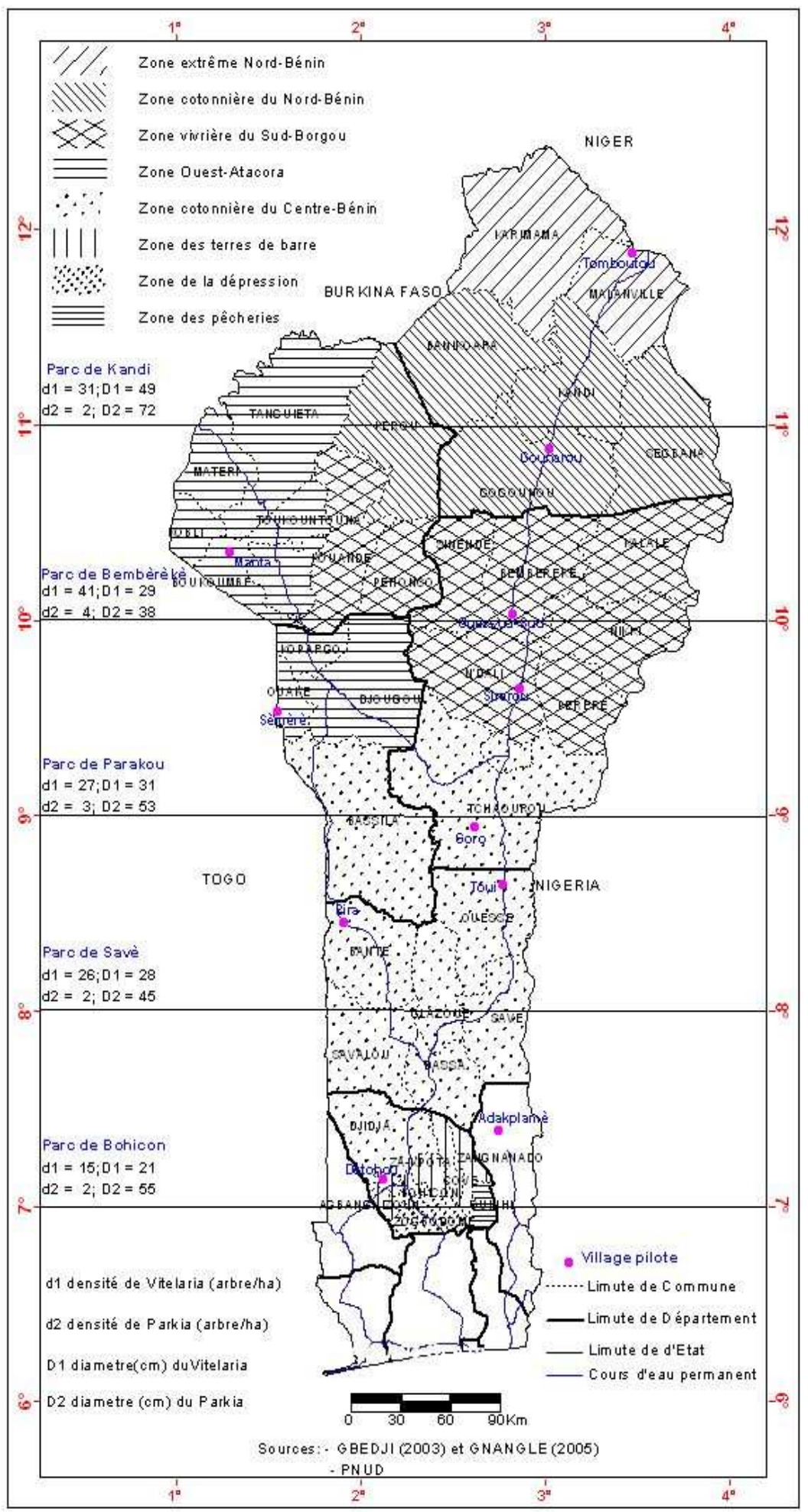

Figure 1 : Localisation des provenances des semences de néré. 
Tableau 1 : Description et symboles des provenances considérées.

\begin{tabular}{|c|c|c|c|c|}
\hline $\begin{array}{l}\text { Code des } \\
\text { provenances }\end{array}$ & Parcs & Communes & Villages & $\begin{array}{l}\text { Coordonnées } \\
\text { géographiques des points } \\
\text { de collecte des graines }\end{array}$ \\
\hline $\mathrm{P} 1$ & \multirow{3}{*}{$\begin{array}{c}\text { Bohicon } \\
\text { (zone guinéenne) }\end{array}$} & Bohicon & $\begin{array}{l}\text { Goukon- } \\
\text { Agnondji }\end{array}$ & $\begin{array}{l}07^{\circ} 14^{\prime} 17 \mathrm{~N} \\
02^{\circ} 03^{\prime} 35 \mathrm{E} \\
\end{array}$ \\
\hline $\mathrm{P} 2$ & & Djidja & Kassèhlo & $\begin{array}{l}07^{\circ} 26^{\prime} 03 \mathrm{~N} \\
02^{\circ} 04^{\prime} 10 \mathrm{E}\end{array}$ \\
\hline P3 & & $\begin{array}{l}\text { Dassa- } \\
\text { Zoumè }\end{array}$ & Yawa & $\begin{array}{l}07^{\circ} 35^{\prime} 16 \mathrm{~N} \\
02^{\circ} 09^{\prime} 39 \mathrm{E}\end{array}$ \\
\hline $\mathrm{P} 4$ & \multirow{4}{*}{$\begin{array}{c}\text { Savè } \\
\text { (zone soudano- } \\
\text { guinéenne) }\end{array}$} & Glazoué & Akpikpi & $\begin{array}{l}07^{\circ} 54^{\prime} 35 \mathrm{~N} \\
02^{\circ} 13^{\prime} 51 \mathrm{E}\end{array}$ \\
\hline P5 & & Savè & $\begin{array}{c}\text { Challa } \\
\text { Gbahungba }\end{array}$ & $\begin{array}{l}08^{\circ} 14^{\prime} 47 \mathrm{~N} \\
02^{\circ} 38^{\prime} 45 \mathrm{E} \\
\end{array}$ \\
\hline P6 & & Ouessè & Malété & $\begin{array}{l}08^{\circ} 44^{\prime} 56 \mathrm{~N} \\
02^{\circ} 35^{\prime} 56 \mathrm{E}\end{array}$ \\
\hline P7 & & Tchaourou & $\begin{array}{c}\text { Tchatchou } \\
\text { (Campement) }\end{array}$ & $\begin{array}{l}09^{\circ} 05^{\prime} 18 \mathrm{~N} \\
02^{\circ} 33^{\prime} 23 \mathrm{E}\end{array}$ \\
\hline P8 & \multirow{3}{*}{$\begin{array}{l}\text { Parakou } \\
\text { (zone soudano- } \\
\text { guinéenne) }\end{array}$} & N'Dali & Komiguéa & $\begin{array}{l}09^{\circ} 26^{\prime} 49 \mathrm{~N} \\
02^{\circ} 37^{\prime} 30 \mathrm{E}\end{array}$ \\
\hline P9 & & Djougou & Yatanfaga & $\begin{array}{l}09^{\circ} 34^{\prime} 20 \mathrm{~N} \\
01^{\circ} 48^{\prime} 45 \mathrm{E}\end{array}$ \\
\hline P10 & & Péhunco & Tobré & $\begin{array}{l}10^{\circ} 11^{\prime} 33 \mathrm{~N} \\
02^{\circ} 08^{\prime} 09 \mathrm{E}\end{array}$ \\
\hline P11 & \multirow{3}{*}{$\begin{array}{l}\text { Bembéréké (zone } \\
\text { soudanienne) }\end{array}$} & Natitingou & Pouya & $\begin{array}{l}10^{\circ} 17^{\prime} 25 \mathrm{~N} \\
01^{\circ} 29^{\prime} 26 \mathrm{E}\end{array}$ \\
\hline $\mathrm{P} 12$ & & Gogounou & Gnindarou & $\begin{array}{l}10^{\circ} 40^{\prime} 25 \mathrm{~N} \\
02^{\circ} 45^{\prime} 46 \mathrm{E}\end{array}$ \\
\hline P13 & & Gounarou & Bantansouè & $\begin{array}{l}10^{\circ} 55^{\prime} 08 \mathrm{~N} \\
02^{\circ} 51^{\prime} 32 \mathrm{E}\end{array}$ \\
\hline P14 & \multirow{2}{*}{$\begin{array}{c}\text { Kandi } \\
\text { (zone } \\
\text { soudanienne) }\end{array}$} & Kandi & Angaradébou & $\begin{array}{l}11^{\circ} 18^{\prime} 59 \mathrm{~N} \\
03^{\circ} 02^{\prime} 16 \mathrm{E} \\
\end{array}$ \\
\hline $\mathrm{P} 15$ & & Malanville & Bangou & $\begin{array}{l}11^{\circ} 38^{\prime} 38 \mathrm{~N} \\
03^{\circ} 11^{\prime} 09 \mathrm{E} \\
\end{array}$ \\
\hline
\end{tabular}

Tableau 2 : Description des traitements d'accélération de croissance des plants de néré appliqués lors de l'essai.

\begin{tabular}{ll}
\hline \multicolumn{1}{c}{ Traitements } & \multicolumn{1}{c}{ Description } \\
\hline $\mathrm{T}_{0}$ & Témoin (sans application d'accélérateur de croissance). \\
\hline $\mathrm{T}_{1}$ & $\begin{array}{l}\text { Application de NPK 1-2-1 à la dose de } 25 \mathrm{~g} / \mathrm{pot} \text {. A l'application, l'engrais a été } \\
\text { fractionné en deux. Le 1/3 de la dose totale, soit } 8 \mathrm{~g} / \text { pot et les } 2 / 3 \text { restant, soit } 17 \\
\text { g/pot ont été appliqués à } 30 \text { jours d'intervalle. }\end{array}$ \\
\hline $\mathrm{T}_{10}$ & $\begin{array}{l}\text { Aspersion foliaire tous les } 10 \text { jours d'un extrait aqueux de feuilles de Moringa } \\
\text { oleifera préparé avec de l'éthanol à } 80 \% \text { puis dilué à 15 \% dans de l'eau. }\end{array}$ \\
\hline $\mathrm{T}_{20}$ & $\begin{array}{l}\text { Aspersion foliaire tous les } 20 \text { jours du même extrait aqueux de feuilles de Moringa } \\
\text { oleifera }\end{array}$ \\
\hline $\mathrm{T}_{30}$ & $\begin{array}{l}\text { Aspersion foliaire tous les } 30 \text { jours de l'extrait aqueux de feuilles de Moringa } \\
\text { oleifera }\end{array}$ \\
\hline
\end{tabular}




\section{RESULTATS}

Effet des provenances et des accélérateurs de croissance sur les paramètres morphologiques des plants de néré

Les valeurs moyennes du diamètre au collet, de la hauteur et du nombre de feuilles des 15 provenances au $140^{\text {ème }}$ jour après semis et selon les traitements de croissance appliqués révèlent qu'en absence d'accélérateur de croissance (T0), la provenance P6 de néré donne les caractéristiques morphologiques les plus élevées $(6,6 \mathrm{~cm}$ de diamètre au collet, $26,3 \mathrm{~cm}$ de hauteur totale et 8,5 feuilles). Les provenances $\mathrm{P} 1, \mathrm{P} 4, \mathrm{P} 5, \mathrm{P} 8, \mathrm{P} 13$ et $\mathrm{P} 14$ donnent des valeurs moyennes de caractéristiques morphologiques alors que $\mathrm{P} 2$, P3, P7, P9, P10, P11 et P12 présentent les valeurs les plus faibles (Tableau 3 ).

Avec l'application d'engrais $\left(T_{1}\right)$, les provenances $\mathrm{P} 1, \mathrm{P} 2, \mathrm{P} 3, \mathrm{P} 4, \mathrm{P} 6, \mathrm{P} 8, \mathrm{P} 10$ et P13 présentent les valeurs les plus élevées pour les caractéristiques morphologiques des plants alors que la provenance P15 présente les valeurs les plus faibles de ces paramètres $(5,5 \mathrm{~cm}$ de diamètre au collet; $15,2 \mathrm{~cm}$ de hauteur et 5,9 feuilles) (Tableau 3).

Les autres provenances donnent des performances moyennes. Par ailleurs, toutes les provenances présentent globalement les valeurs les plus élevées des caractéristiques morphologiques pour l'angrais NPK. Parmi les traitements liés à l'application d'extraits aqueux de feuilles de $M$. oleifera, celui relatif à l'application tous les 10 jours de l'extrait aqueux présente des valeurs relativement plus élevées des caractéristiques morphologiques de la plupart des provenances. Les autres traitements présentent des valeurs proches de celles du témoin T0. Avec l'application d'extraits aqueux de $M$. oleifera tous les 10 jours, les provenances P1, P2, P6 et P8 ont les valeurs les plus élevées des caractéristiques morphologiques alors que les provenances $\mathrm{P} 3$, $\mathrm{P} 7, \mathrm{P} 12, \mathrm{P} 13, \mathrm{P} 14$ et $\mathrm{P} 15$ présentent des plants de petites tailles.

Par ailleurs, quelles que soient les provenances, on note une corrélation positive entre les trois paramètres morphologiques et ceci, indépendamment des traitements appliqués. Le coefficient de corrélation entre la hauteur totale des plantules et le diamètre au collet est très hautement significatif $(0,644)$. Le coefficient de corrélation entre la hauteur totale et le nombre de feuilles est aussi très hautement significatif $(0,310)$. Seule la corrélation entre le diamètre au collet et le nombre de feuilles est juste significative $(0,069)$. Ces résultats indiquent une synergie de croissance entre les paramètres morphologiques considérés pour $P$. biglobosa, surtout entre le diamètre au collet et la hauteur totale.

\section{Effet des provenances et traitements sur la croissance des plants de néré}

Les résultats d'analyse de la variance sur mesures répétées effectuées pour comparer d'une part les provenances et d'autre part les traitements du point de vue de la croissance des plants de néré indiquent globalement un effet très hautement significatif du temps sur l'évolution des trois paramètres (diamètre au collet, hauteur et nombre de feuilles). Il y en est globalement de même de l'effet des autres facteurs et de leurs interactions sur les paramètres morphologiques au $140^{\text {ème }}$ jour après semis (Tableau 4).

De ce fait, le nombre de jours après semis influence les paramètres de même que la comparaison des provenances et des traitements. Pour décrire et analyser l'effet du temps sur les caractéristiques morphologiques des provenances et l'effet des traitements, les coefficients de régression $b_{0}, b_{1}$ et $b_{2}$ des équations (1) et (2) sont soumises à l'analyse de la variance à deux facteurs: provenance et traitement. Les résultats sont présentés au Tableau 5 et indiquent pour les paramètres de croissance du diamètre un effet significatif des traitements, des provenances et aussi de leur interaction. Ceci implique alors que les traitements n'induisent pas le même diamètre initial et la même vitesse de croissance en diamètre des plants de néré. De même, les provenances ne présentent pas aussi le même 
diamètre initial et la même vitesse de croissance en diamètre des plants. En ce qui concerne la hauteur et le nombre de feuilles, la même tendance que pour le diamètre a été observée, excepté le fait que les traitements n'ont pas d'effet significatif sur ceux-ci.

La comparaison des provenances suivant les coefficients de croissance $b_{0}$ et $b_{1}$ en fonction des traitements (Tableau 6) révèle que, de façon générale, les vitesses exponentielles de croissance du diamètre et aussi de la hauteur, présentent les valeurs les plus élevées avec l'application d'engrais (T1). Avec ce traitement, les valeurs initiales du diamètre et de la hauteur sont globalement parmi les plus faibles. En ce qui concerne le nombre de feuilles, la Figure 2 indique une croissance très rapide du nombre de feuilles des plants sous application d'engrais NPK avec toutefois une chute brusque de ce même paramètre à partir du $110^{\text {ème }}$ jour après semis et ceci pour la plupart des groupes de semences excepté G3.

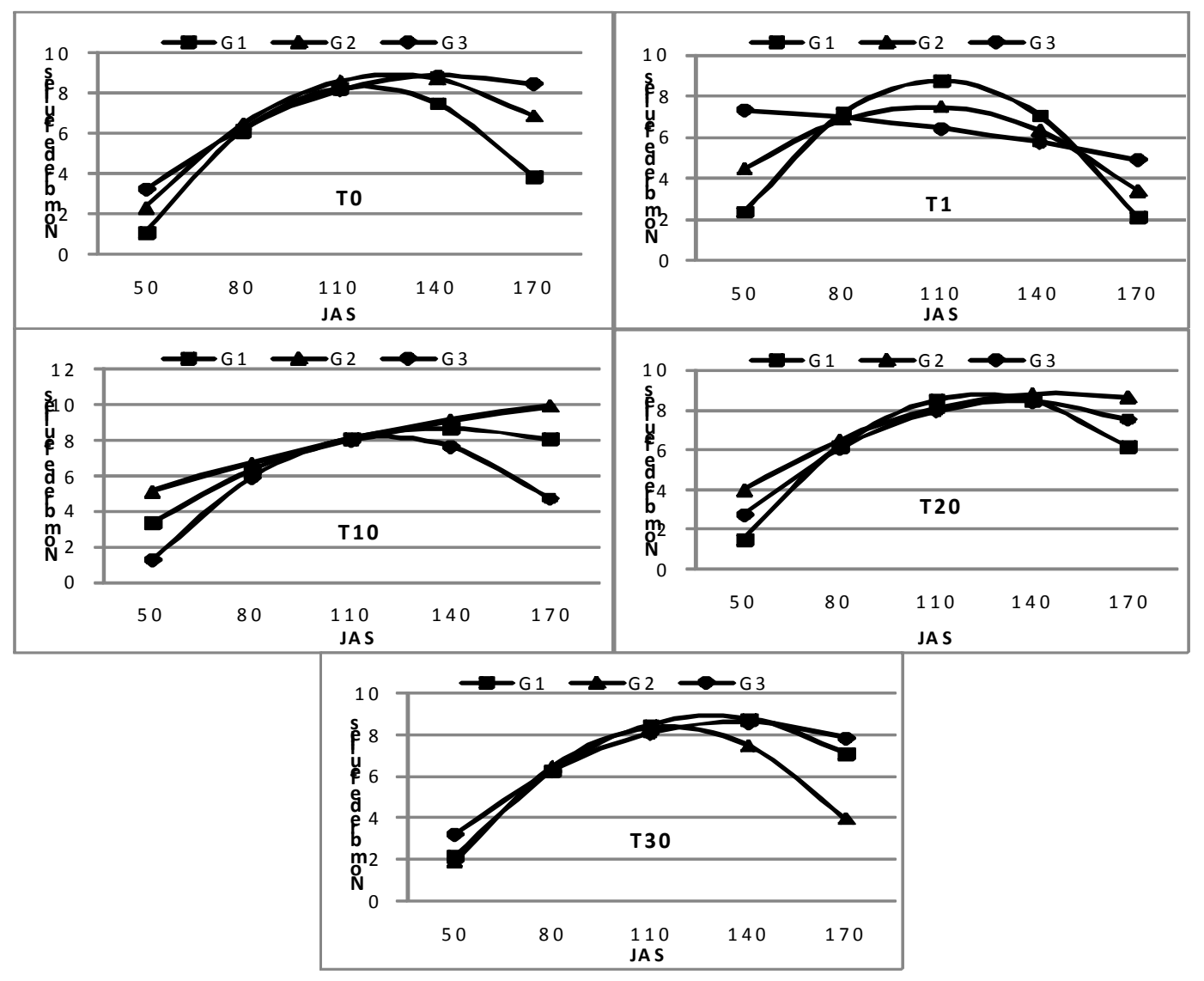

Figure 2 : Evolution du nombre de feuilles en fonction des traitements et groupes de provenances.

JAS : jours après semis, T0 : sans accélérateur de croissance; T1 : engrais NPK ;

T10 : extrait aqueux de $M$. oleifera tous les 10 jours ; T20 : extrait aqueux de $M$. oleifera tous les 20 jours ;

T30 : extrait aqueux de $M$. oleifera tous les 30 jours.

T0 : G1=P1, P2, P3, P12 ; G2=P4, P6, P7, P9, P14 ; G3=P5, P8, P10, P11, P13, P15.

T1 : G1=P1, P2, P3, P4, P6, P8, P13; G2=P5, P7, P9, P10, P11, P12 ; G3=P14, P15.

T10 : G1=P1, P2, P5, P13, P14; G2=P3, P10; G3=P4, P6, P7, P9, P8, P11, P12, P15.

T20 : G1=P1, P5, P7, P10, P11 ; G2=P2, P3, P4, P12, P13, P14, P15 ; G3=P6, P8, P9.

T30 : G1=P1, P2, P4, P7 ; G2=P3, P10 ; G3=P5, P6, P8, P9, P11, P12, P13, P14, P15. 
Tableau 3 : Paramètres morphologiques des provenances de néré à 140 jours après semis suivant les traitements appliqués.

\begin{tabular}{|c|c|c|c|c|c|c|c|c|c|c|c|c|c|c|c|}
\hline \multirow{2}{*}{ Prov } & \multicolumn{3}{|c|}{ T0 } & \multicolumn{3}{|c|}{ T1 } & \multicolumn{3}{|c|}{ T10 } & \multicolumn{3}{|c|}{ T20 } & \multicolumn{3}{|c|}{ T30 } \\
\hline & DC & Ht & NF & DC & $\mathbf{H t}$ & NF & DC & Ht & NF & DC & Ht & NF & DC & Ht & NF \\
\hline P1 & $5,6^{\mathrm{a}}$ & $19,6^{\mathrm{a}}$ & $8,4^{\mathrm{a}}$ & $7,9^{\mathrm{a}}$ & $25,8^{\mathrm{a}}$ & $6,6^{\mathrm{a}}$ & $5,6^{\mathrm{a}}$ & $19,8^{\mathrm{a}}$ & $8,6^{\mathrm{a}}$ & $5,9^{\mathrm{a}}$ & $22,9^{\mathrm{a}}$ & $9,1^{\mathrm{a}}$ & $5,7^{\mathrm{a}}$ & $19,1^{\mathrm{a}}$ & $8,7^{\mathrm{a}}$ \\
\hline $\mathrm{P} 2$ & $4,9^{\mathrm{b}}$ & $17,3^{\mathrm{b}}$ & $7,5^{\mathrm{b}}$ & $7,5^{\mathrm{a}}$ & $24,3^{\mathrm{b}}$ & $7,9^{\mathrm{a}}$ & $5,6^{\mathrm{a}}$ & $20,8^{\mathrm{a}}$ & $9,1^{\mathrm{a}}$ & $6,1^{\mathrm{a}}$ & $21,1^{\mathrm{a}}$ & $8,8^{\mathrm{a}}$ & $5,8^{\mathrm{a}}$ & $19,7^{\mathrm{a}}$ & $8,8^{\mathrm{a}}$ \\
\hline P3 & $5,4^{\mathrm{b}}$ & $16,9^{\mathrm{b}}$ & $8,1^{\mathrm{b}}$ & $8,8^{\mathrm{a}}$ & $25,2^{\mathrm{a}}$ & $5,9^{\mathrm{a}}$ & $5,8^{\mathrm{b}}$ & $17,3^{\mathrm{b}}$ & $8,8^{\mathrm{b}}$ & $5,6^{\mathrm{b}}$ & $17,0^{\mathrm{b}}$ & $8,8^{\mathrm{b}}$ & $5,9^{\mathrm{b}}$ & $16,3^{\mathrm{b}}$ & $8,1^{\mathrm{b}}$ \\
\hline P4 & $5,5^{\mathrm{a}}$ & $20,4^{\mathrm{a}}$ & $9,4^{\mathrm{a}}$ & $9,1^{\mathrm{a}}$ & $25,5^{\mathrm{a}}$ & $7,6^{\mathrm{a}}$ & $5,1^{\mathrm{c}}$ & $18,4^{\mathrm{c}}$ & $8,9^{c}$ & $5,3^{\mathrm{c}}$ & $19,6^{\mathrm{c}}$ & $9,1^{\mathrm{c}}$ & $4,9^{\mathrm{a}}$ & $19,7^{\mathrm{a}}$ & $8,7^{\mathrm{a}}$ \\
\hline P5 & $5,7^{\mathrm{a}}$ & $20,1^{\mathrm{a}}$ & $8,8^{\mathrm{a}}$ & $7,5^{\mathrm{b}}$ & $19,6^{\mathrm{b}}$ & $6,2^{\mathrm{b}}$ & $5,0^{\mathrm{c}}$ & $19,2^{\mathrm{c}}$ & $8,8^{\mathrm{c}}$ & $5,1^{\mathrm{b}}$ & $17,3^{\mathrm{b}}$ & $8,0^{\mathrm{b}}$ & $5,6^{\mathrm{a}}$ & $18,6^{\mathrm{a}}$ & $8,6^{\mathrm{a}}$ \\
\hline P6 & $6,6^{\mathrm{c}}$ & $26,3^{c}$ & $8,5^{\mathrm{c}}$ & $8,0^{\mathrm{a}}$ & $27,1^{\mathrm{a}}$ & $7,7^{\mathrm{a}}$ & $5,7^{\mathrm{a}}$ & $21,2^{\mathrm{a}}$ & $8,2^{\mathrm{a}}$ & $5,7^{\mathrm{a}}$ & $20,7^{\mathrm{a}}$ & $8,7^{\mathrm{a}}$ & $4,8^{\mathrm{b}}$ & $17,6^{\mathrm{b}}$ & $6,9^{\mathrm{b}}$ \\
\hline P7 & $5,1^{\mathrm{b}}$ & $16,7^{\mathrm{b}}$ & $8,5^{\mathrm{b}}$ & $8,2^{\mathrm{b}}$ & $19,3^{\mathrm{b}}$ & $6,3^{\mathrm{b}}$ & $5,5^{\mathrm{b}}$ & $15,4^{\mathrm{b}}$ & $8,4^{\mathrm{b}}$ & $5,1^{\mathrm{b}}$ & $15,3^{\mathrm{b}}$ & $7,8^{\mathrm{b}}$ & $5,4^{\mathrm{b}}$ & $15,5^{\mathrm{b}}$ & $8,6^{\mathrm{b}}$ \\
\hline P8 & $5,4^{\mathrm{a}}$ & $22,3^{\mathrm{a}}$ & $9,3^{\mathrm{a}}$ & $8,3^{\mathrm{a}}$ & $24,1^{\mathrm{a}}$ & $7,1^{\mathrm{a}}$ & $5,5^{\mathrm{a}}$ & $20,3^{a}$ & $8,5^{\mathrm{a}}$ & $5,2^{\mathrm{c}}$ & $20,1^{\mathrm{c}}$ & $8,8^{\mathrm{c}}$ & $4,6^{\mathrm{b}}$ & $17,2^{\mathrm{b}}$ & $8,7^{\mathrm{b}}$ \\
\hline P9 & $5,1^{\mathrm{b}}$ & $17,3^{\mathrm{b}}$ & $8,0^{\mathrm{b}}$ & $6,8^{\mathrm{b}}$ & $20,4^{\mathrm{b}}$ & $5,7^{\mathrm{b}}$ & $5,7^{\mathrm{c}}$ & $18,6^{\mathrm{c}}$ & $8,4^{\mathrm{c}}$ & $4,9^{\mathrm{b}}$ & $16,6^{b}$ & $7,8^{\mathrm{b}}$ & $5,6^{\mathrm{b}}$ & $18,0^{\mathrm{b}}$ & $8,3^{\mathrm{b}}$ \\
\hline P10 & $5,6^{\mathrm{b}}$ & $17,3^{\mathrm{b}}$ & $8,1^{\mathrm{b}}$ & $8,6^{\mathrm{a}}$ & $23,8^{\mathrm{a}}$ & $7,2^{\mathrm{a}}$ & $5,3^{\mathrm{c}}$ & $19,1^{\mathrm{c}}$ & $9,3^{\mathrm{c}}$ & $5,6^{\mathrm{c}}$ & $18,2^{\mathrm{c}}$ & $7,9^{\mathrm{c}}$ & $5,4^{\mathrm{b}}$ & $16,7^{\mathrm{b}}$ & $8,1^{\mathrm{b}}$ \\
\hline P11 & $5,1^{\mathrm{b}}$ & $16,5^{\mathrm{b}}$ & $8,1^{\mathrm{b}}$ & $6,4^{\mathrm{b}}$ & $18,3^{\mathrm{b}}$ & $5,5^{\mathrm{b}}$ & $5,8^{\mathrm{c}}$ & $19,0^{\mathrm{c}}$ & $9,2^{\mathrm{c}}$ & $5,4^{\mathrm{b}}$ & $17,5^{\mathrm{b}}$ & $9,2^{\mathrm{b}}$ & $5,5^{\mathrm{a}}$ & $19,8^{\mathrm{a}}$ & $10,1^{\mathrm{a}}$ \\
\hline P12 & $4,3^{\mathrm{b}}$ & $16,3^{\mathrm{b}}$ & $8,6^{\mathrm{b}}$ & $7,3^{\mathrm{b}}$ & $21,1^{\mathrm{b}}$ & $6,1^{\mathrm{b}}$ & $4,7^{\mathrm{b}}$ & $16,5^{\mathrm{b}}$ & $7,9^{\mathrm{b}}$ & $4,7^{\mathrm{b}}$ & $16,7^{\mathrm{b}}$ & $8,2^{\mathrm{b}}$ & $4,6^{\mathrm{b}}$ & $16,6^{\mathrm{a}}$ & $8,3^{\mathrm{b}}$ \\
\hline P13 & $4,6^{\mathrm{a}}$ & $19,4^{\mathrm{a}}$ & $8,8^{\mathrm{a}}$ & $7,4^{\mathrm{a}}$ & $22,9^{\mathrm{a}}$ & $6,3^{\mathrm{a}}$ & $4,8^{\mathrm{b}}$ & $16,7^{\mathrm{b}}$ & $8,1^{\mathrm{b}}$ & $5,1^{\mathrm{c}}$ & $18,8^{\mathrm{c}}$ & $9,3^{\mathrm{c}}$ & $4,8^{\mathrm{b}}$ & $17,9^{\mathrm{a}}$ & $8,2^{\mathrm{b}}$ \\
\hline P14 & $5,4^{\mathrm{a}}$ & $19,3^{\mathrm{a}}$ & $8,7^{\mathrm{a}}$ & $6,7^{\mathrm{b}}$ & $18,7^{\mathrm{b}}$ & $6,5^{\mathrm{b}}$ & $4,9^{\mathrm{b}}$ & $16,1^{\mathrm{b}}$ & $7,9^{\mathrm{b}}$ & $5,1^{\mathrm{c}}$ & $18,5^{\mathrm{c}}$ & $8,2^{c}$ & $4,9^{\mathrm{b}}$ & $16,6^{\mathrm{a}}$ & $8,2^{\mathrm{b}}$ \\
\hline P15 & $4,5^{\mathrm{b}}$ & $14,2^{\mathrm{b}}$ & $8,7^{\mathrm{b}}$ & $5,5^{\mathrm{c}}$ & $15,2^{\mathrm{c}}$ & $5,9^{\mathrm{c}}$ & $4,5^{\mathrm{b}}$ & $15,3^{\mathrm{b}}$ & $8,3^{\mathrm{b}}$ & $4,3^{\mathrm{b}}$ & $15,3^{b}$ & $7,7^{\mathrm{a}}$ & $4,1^{\mathrm{c}}$ & $13,8^{\mathrm{c}}$ & $8,4^{\mathrm{c}}$ \\
\hline
\end{tabular}

Prov: provenance; DC: diamètre au collet $(\mathrm{mm})$; $\mathrm{Ht}$ : hauteur $(\mathrm{m})$; NF : Nombre de feuilles ; Pour un traitement et un paramètre morphologique donnés, les valeurs de même lettre ne sont pas significativement différents (classification numérique avec la méthode de Ward).

Tableau 4 : Résultats d'analyse de variance sur les mesures répétées: modèle fixe à 2 facteurs (provenance et traitement): valeurs de $\mathrm{F}$ et niveaux de significativité.

\begin{tabular}{|c|c|c|c|c|}
\hline Source & DF & Diamètre & Hauteur & Nombre de Feuilles \\
\hline Temps & $3(2)$ & $5387,9^{\text {****** }}$ & $4262,3^{* * * *}$ & $921,8^{* * * *}$ \\
\hline Provenance $(\mathrm{P})$ & $14(14)$ & $18,0^{* * *}$ & $17,2^{* * *}$ & $7,8^{* * *}$ \\
\hline Temps x P & $42(28)$ & $7,6^{* * *}$ & $9,2^{* * *}$ & $4,2^{* * *}$ \\
\hline Traitement $(\mathrm{Tt})$ & $4(4)$ & $265,1^{* * *}$ & $42,4^{* * *}$ & $11,1^{* * *}$ \\
\hline Temps x Tt & $12(8)$ & $121,6^{* * *}$ & $19,6^{* * *}$ & $60,8^{* * *}$ \\
\hline $\mathrm{P} \times \mathrm{T}$ & $56(56)$ & $2,1^{* * * *}$ & $1,5^{*}$ & $1,6^{* *}$ \\
\hline Temps x P x T & $168(112)$ & $2,5^{* * *}$ & $2,0^{* * *}$ & $2,1^{* * *}$ \\
\hline
\end{tabular}
parenthèses sont relatifs au paramètre «nombre de feuilles » mesuré à 3 dates contrairement au diamètre et à la hauteur mesurés à 4 dates.

En effet, les provenances P14 et P15 constituant ce groupe présente une allure pratiquement linéaire décroissante du nombre de feuilles à partir de 50 jours après semis.

Parmi les traitements à base d'extraits aqueux de $M$. oleifera, celui relatif à l'application tous les 10 jours de l'extrait aqueux présente une croissance morphologique significative des organes des jeunes plants et surtout un accroissement régulier du nombre de feuilles de l'espèce avec une chute relativement moins prononcée 
Tableau 5 : Résultats d'analyse de variance sur les coefficients du modèle de croissance des paramètres morphologiques du néré: valeurs de $\mathrm{F}$ et niveaux de significativité.

\begin{tabular}{|c|c|c|c|c|c|c|c|c|}
\hline \multirow{2}{*}{ Source } & \multicolumn{3}{|c|}{ Diamètre (cm) } & \multicolumn{2}{|c|}{ Hauteur (cm) } & \multicolumn{3}{|c|}{ Nombre de feuilles } \\
\hline & DF & $b_{0}$ & $b_{1}$ & $b_{0}$ & $b_{1}$ & $b_{0}$ & $b_{2}$ & $b_{2}$ \\
\hline Traitement $(\mathrm{T})$ & 4 & $4,2 * *$ & $79,8 * * *$ & $0,4 \mathrm{~ns}$ & $8,1 * * *$ & $1,1 \mathrm{~ns}$ & $1,3 \mathrm{~ns}$ & $3,6^{* *}$ \\
\hline Provenance $(\mathrm{P})$ & 14 & $7,5 * * *$ & $4,2 * * *$ & $13,6 * * *$ & $8,9 * * *$ & $2,3 * *$ & $2,3 * *$ & $2,3 * *$ \\
\hline $\mathrm{T} \times \mathrm{P}$ & 56 & $2,0 * * *$ & $2,0 * * *$ & $2,2 * * *$ & $1,8 * * *$ & $2,1 * * *$ & $2,2 * * *$ & $2,2 * * *$ \\
\hline
\end{tabular}

Tableau 6: Valeurs moyennes des coefficients $b_{0}, b_{1}$ et $b_{2}$ des modèles de croissance du diamètre au collet et de la hauteur des groupes de provenances de néré suivant les traitements.

\begin{tabular}{llccccc}
\hline \multicolumn{5}{c}{ Diamètre } & \multicolumn{5}{c}{ Hauteur (cm) } \\
\hline Groupe & $\begin{array}{l}\text { Moyenne } \\
(\mathbf{c m})\end{array}$ & $b_{\mathbf{0}}$ & $b_{\mathbf{1}}$ & $\begin{array}{l}\text { Moyenne } \\
(\mathbf{c m})\end{array}$ & $b_{\mathbf{o}}$ & $b_{\mathbf{1}}$ \\
\hline T0 & & & & & & \\
G1 & 5,4 & 1,85 & 0,0074 & 17,8 & 4,91 & 0,0098 \\
G2 & 5,4 & 1,96 & 0,0075 & 20,4 & 5,38 & 0,0095 \\
G3 & 5,2 & 1,94 & 0,0071 & 18,3 & 5,49 & 0,0089 \\
T1 & & & & & & \\
G1 & 7,6 & 1,71 & 0,0116 & 23,2 & 5,09 & 0,0116 \\
G2 & 8,1 & 1,84 & 0,0105 & 21,8 & 5,30 & 0,0100 \\
G3 & 7,6 & 1,96 & 0,0091 & 19,3 & 5,16 & 0,0070 \\
T10 & & & & & & \\
G1 & 5,4 & 2,00 & 0,0069 & 18,9 & 5,48 & 0,0091 \\
G2 & 5,2 & 1,95 & 0,0077 & 17,8 & 5,94 & 0,0081 \\
G3 & 5,2 & 1,84 & 0,0076 & 18,5 & 4,97 & 0,0096 \\
T20 & & & & & & \\
G1 & 5,3 & 1,83 & 0,0078 & 19,0 & 5,14 & 0,0091 \\
G2 & 5,2 & 1,91 & 0,0073 & 17,8 & 5,51 & 0,0089 \\
G3 & 5,2 & 1,90 & 0,0073 & 18,6 & 5,32 & 0,0092 \\
T30 & & & & & & \\
G1 & 5,4 & 1,83 & 0,0079 & 17,7 & 5,03 & 0,0093 \\
G2 & 5,8 & 1,89 & 0,0079 & 17,4 & 5,91 & 0,0075 \\
G3 & 5,0 & 1,86 & 0,0073 & 17,5 & 5,47 & 0,0087 \\
\hline & & & & & &
\end{tabular}

à partir du $110^{\text {ème }}$ jour après semis. Dans le cas d'application de ce traitement, le groupe G2 constitué de P2, P3, P4, P12, P13, P14 et P15 présente le nombre le plus élevé de feuilles au départ et un accroissement relativement rapide du nombre de feuilles suivi d'une stabilité relative à partir du $130^{\text {ème }}$ jour après semis.

\section{DISCUSSION}

La domestication de la plupart des arbres fruitiers sauvages comme le néré 
(Parkia biglobosa Jack, R. Br.) nécessite l'identification des meilleures provenances ou individus élites de l'espèce et des conditions optimales de croissance. La présente étude a exploré et identifié les meilleures provenances en termes de croissance morphologique des jeunes plants et l'effet de cinq différents stimulateurs de croissance sur les jeunes plants.

Effet des provenances sur la morphologie et la croissance des plants de néré

Les résultats de l'étude ont indiqué des différences significatives entre les provenances de néré en ce qui concerne les caractéristiques morphologiques des jeunes plants en pépinière. Avec l'application d'engrais, la provenance a donné les meilleures performances mais avec d'autres provenances comme P1, P2, P3, P4, P8, P10 et $\mathrm{P} 13$, appartenant aux différentes zones climatiques du Bénin. Bien que certaines études aient indiqué l'effet du climat et du gradient écologique sur la morphologie des plants (Soloviev et al., 2004; Maranz et Wiesman, 2003; Giordano, 1972), les résultats obtenus de cette étude ne semblent pas révéler l'impact de la variabilité climatique sur la morphologie des jeunes plants. En fait, les différences phénotypiques entre les provenances de néré pourraient plutôt être expliquées par des différences au niveau micro-environnemental entre les lieux de collecte des semences. Des facteurs comme le sol, l'âge et les caractéristiques génétiques des arbres-mères d'où proviennent les semences utilisées seraient à l'origine de ces différences de morphologie (Salazar et Quesada, 1987; Assogbadjo et al., 2005, 2006). Des tests de croissance basés sur des graines provenant d'arbres-élites, à travers une sélection participative, donneraient alors des résultats plus concluants.

La croissance des jeunes plants de néré avec ou sans application de stimulateur de croissance varie aussi en fonction des provenances. La zone climatique d'origine ne semble pas avoir d'impact sur la croissance des jeunes plants, confirmant ainsi un effet significatif de l'âge et de la génétique des arbres-mères (Dianda et Chalifour, 2002).

Effet des traitements sur la morphologie et la croissance des plants de néré

Les accélérateurs de croissance ont un effet significatif sur les jeunes plants du néré. L'utilisation de l'engrais comme stimulateur de croissance a donné de meilleurs résultats tant sur le diamètre au collet des jeunes plants que sur la hauteur et le nombre de feuilles. En général, l'utilisation des extraits aqueux de $M$. oleifera n'a pas donné des résultats meilleurs au témoin T0. Ces résultats indiquent alors que la croissance relativement lente de $P$. biglobosa (Sambe et al., 2010) peut être en partie due à des déficiences azotées du sol de son aire naturelle de distribution. Un apport insuffisant en azote au sol limiterait la croissance des jeunes plants de l'espèce en milieu naturel (Dianda et al., 2009). L'effet de la carence en azote des sols tropicaux sur les arbres en milieu naturel est plus perceptible que celle d'autres nutriments comme le phosphore (Elliot et al., 1994; Davidson et al., 2004). La croissance des arbres dans les forêts amazoniennes par exemple, est plus sensible à la disponibilité de l'azote dans le sol que du phosphore malgré une déficience en phosphore du sol dans cette région (Davidson et al., 2004). Ceci explique que, parmi les traitements à base de $M$. oleifera, seule une application régulière et fréquente (tous les 10 jours) de l'extrait aqueux permettait d'enregistrer une croissance plus significative des jeunes plants qu'en absence de traitement.

Toutefois, ces résultats sont influencés par la provenance des plants de néré. La réponse des jeunes plants à l'application d'engrais n'est donc pas la même selon les provenances, comme l'avait noté Dianda et 
Chalifour (2002) sur les plants de Faidherbia albida.

\section{Conclusion}

La présente étude avait pour objectif d'identifier les meilleures provenances de néré et de tester l'effet de différents accélérateurs de croissance sur de jeunes plants en pépinière. Les provenances de néré testées ont présenté des résultats assez variés et non influencés par les conditions climatiques des régions de prélèvement des semences. Ceci indique la nécessité d'un test de provenance basé sur les graines d'arbres élites pour mieux capturer la variabilité phénotypique des plants. Par ailleurs, l'utilisation d'engrais en sylviculture, bien que relativement récente donne des résultats meilleurs. Cette étude a révélé qu'une utilisation rationnelle de ce fertilisant stimule significativement la croissance de jeunes plants de néré. En dehors de leur effet favorable sur la production de bois, les engrais rendent les plants plus vigoureux et plus résistants aux attaques de certains insectes, bactéries et champignons. Toutefois, les résultats de l'étude ont indiqué qu'une application, tous les 10 jours, d'extraits aqueux de $M$. oleifera peut constituer une alternative biologique à l'engrais minérale.

\section{REFERENCES}

Agbahungba G, Depommier D. 1989. Aspects du parc à karités-nérés (Vitellaria paradoxa Gaertn.f, Parkia biglobosa Jacq) dans le sud du Borgou. Bois et Forêts des Tropiques, 222 (4è trimestre): 41-54.

Assogbadjo AE, Kyndt T, Sinsin B, Gheysen G, Van Damme P. 2006. Patterns of genetic and morphometric diversity in baobab (Adansonia digitata L.) populations across different climatic zones of Benin (West Africa). Annals of Botany, 97: 819-830.
Assogbadjo AE, Sinsin B, Codjia JTC, Van Damme P. 2005. Ecological diversity and pulp, seed and kernel production of the baobab (Adansonia digitata) in Benin. Belgian Journal of Botany. 138(1): 47-56.

CIRAD-GRET-MAE. 2002. Mémento de l'agronome, p. 1691.Codjia JTC, FontonKiki B, Assogbadjo AE, Ekue MRM. 2001. Le baobab (Adansonia digitata), une espèce à usages multiples au Bénin. CECODI / CBDD / Veco / SNV/ FSA, Cotonou, Bénin, p. 45.

Crowder MJ, Hand DJ. 1990. Analysis of Repeated Measures. Chapman and Hall: New York.

Dagnelie P. 1998. Statistique Théorique et Appliquée (vol. 1 \& 2). De Boeck et Larcier: Paris.

Danthu P, Roussel J, Gaye A, El Mazzoudi EH. 1995. Baobab (Adansonia digitata L.) seed pretreatments for germination improvement. Seed Sc. Technol., 23: 469475.

Davidson EA, Carvalho CR, Vieira I, Figueiredo R, Moutinho P, Ishida FY, dos Santos MTP, Guerrero B, Kalif K, Sabá RT. 2004. Nitrogen and phosphorus limitation of biomass growth in a tropical secondary forest. Ecological Applications, 1: 150-163.

Dianda M, Bayala J, Diop T, Ouédraogo SJ. 2009. Improving growth of shea butter tree (Vitellaria paradoxa C.F.Gaertn.) seedlings using mineral $\mathrm{N}, \mathrm{P}$ and arbuscular mycorrhizal (AM) fungi. Biotechnol. Agron. Soc. Environ., 13(1) : 93-102.

Dianda M, Chalifour FP. 2002. Effets du N minéral et du génotype de la plante sur la croissance et la nodulation de Faidherbia albida. Can. J. Bot., 80(3): 241-254.

Dupriez H, De Leener P. 1993. Arbres et agricultures multi étagées d'Afrique. Terres et Vie : 164-168. 
Elliott KJ, White AS. 1994. Effects of light, nitrogen and phosphorus on red pine seedling growth and nutrient use efficiency. Forest Science, 40: 47-58.

Foidl N, Makkar HPS, Becker K. 2001. Potentiel de Moringa oleifera en agriculture et dans l'industrie : Atelier sur Potentiel de développement des produits $d u$ Moringa. Dar es Salaam, Tanzanie, p. 20.

Gbédji EKY. 2003. Caractérisation morphologique et structurale des parcs à néré [Parkia biglobosa (Jack.) R. Br. Ex. G. Don.] au Bénin. Diplôme d'Ingénieur Agronome, Université d'Abomey-Calavi, p. 105.

Giordano E. 1972. Interaction de la sélection et de la culture intensive. Unasylva, 97 98: $82-88$.

Gijsbers HJM, Kessler JJ, Knevel MK. 1994. Dynamics and natural regeneration of woody species in farmed parklands in the Sahel region (Province of Passore, Burkina Faso). Forest Ecol. Manage., 64: 1-12.

Gnanglè CP. 2005. Parcs à karité (Vitellaria paradoxa Gaertner. F.) (Sapotaceae) au Bénin: Importance socio-économique, caractérisation morphologique et structurale, et régénération naturelle. Mémoire de DEA. Université d'AbomeyCalavi, p. 134.

Gutierrez ML. 2000. Production et commercialisation de l'afitin fon dans la région d'Abomey-Bohicon au Bénin. Un exemple d'intégration des femmes à la filière du néré. Montpellier CNEARC, CIRAD, p. 124.

IPGRI. 1999. Vers une approche régionale des ressources génétiques forestières en Afrique sub-saharienne. Recensement Général de la Population et de l'Habitat Sahélien. Actes du premier atelier régional de formation sur la conservation et l'utilisation durable des ressources génétiques forestières en Afrique de l'ouest, Afrique forestière. CIRAD / GILLSS /CORAF/ CTA / DESC / FAO / UNEP / UNSO, Ouagadougou, Burkina Faso, p. 229.

Koura K. 2005. Contribution à l'étude ethnobotanique du néré [Parkia biglobosa (Jacq.) R. Br. ex G. Don] dans les départements de l'Atacora et de la Donga: aspects socioculturels. Atelier Scientifique National, INRAB. AbomeyCalavi, Bénin : 786-799.

Leakey RRB, Pate K, Lombard C. 2005. Domestication potential of Marula (Sclerocarya birrea subsp. caffra) in South Africa and Namibia: Phenotypic variation in nut and kernel traits. Agrofor. Syst., 64: 37-49

Lejoly J. 1990. Liste de 1124 espèces médécinales utilisées dans 11 pays avec indication de la maladie soignée et du nombre de recettes par pays. Projet pharmacopée. ACCT. Labo.Bot. Syst. et Phytosoc. Univ. Libre de Bruxelles, p. 122.

Malaisse F. 1997. Se nourrir en forêt claire africaine. Approche écologique et nutritionnelle, CTA: Wageningen.

Maranz S, Wiesman Z. 2003. Evidence for indigenous selection and distribution of the shea tree, Vitellaria paradoxa, and its potential significance to prevailing parkland savanna tree patterns in subSaharan Africa north of the equator. $J$. Biogeogr., 30 : 1505-1516.

Ouédraogo AS. 1995. Parkia biglobosa (Leguminosae) en Afrique de l'Ouest: Biosystématique et Amélioration. These de doctorat, Wageningen University, Institute for Forestry and Nature Research, IBN-DLO, Wageningen, p. 205.

Salazar R, Quesada M. 1987. Provenance variation in Guazuma ulmifolia L. in 
Costa Rica. Commonwealth Forestry Review, 66: 317-324.

Sambe MAN, Sagna M, Sy MO. 2010. Seed germination and in vitro plant regeneration of Parkia biglobosa (Jacq.) Benth. African Journal of Biotechnology, 9(21): 3099-3108.

SAS Institute Inc. 2003. SAS Online Doc® 9.1. Cary, NC: SAS Institute Inc.

Sokpon N. 1994. Tenure foncière et propriété des ligneux dans les systèmes agroforestiers traditionnels au Bénin. Ann. Fac. SC. Kisangani., ${ }^{\circ}$ spéc. : 115122.
Sokpon N, Ouinsavi C. 2004. Contribution à l'étude ethnobotanique du néré [Parkia biglobosa (Jacq.) R. BR. ex G. Don] dans les départements du Zou et des Collines : aspects socioculturels. Atelier Scientifique National, INRAB. Abomey-Calavi, Bénin, p. 57.

Soloviev P, Niang TD, Gaye A, Totte A. 2004. Variabilité des caractères physicochimiques des fruits de trois espèces ligneuses de cueillette, récoltés au Sénégal: Adansonia digitata, Balanites aegyptiaca et Tamarindus indica. Fruits, 59 : 109-119. 